\title{
Performance Analysis of Asynchronous ABSF Configuration in Large-Scale Femtocell Networks
}

\author{
(Invited Paper) \\ He Wang*, Mark C. Reed ${ }^{* \dagger}$, Xiangyun Zhou ${ }^{\dagger}$, and Wei Bai ${ }^{\ddagger}$ \\ *University of New South Wales (UNSW) Canberra, ACT 2600, Australia \\ ${ }^{\dagger}$ Research School of Engineering, the Australian National University, ACT 0200, Australia \\ ${ }_{\ddagger}^{\ddagger}$ SONY Corporation, Beijing, China \\ Email: mark.reed@unsw.edu.au
}

\begin{abstract}
This paper investigates a combined macro and femtocell communications deployment where macro users suffer significant downlink interference from femtocells. Based upon the large-scale cellular network model using stochastic geometry, we analyze the performance of the asynchronous ABSF configuration, where femtocell independently reserves resource for the victim macro user based on the condition of intercell interference. The trade-off between reducing this interference (and thus improving coverage probability) versus maximising the available femtocell resources (overall network throughput) has been demonstrated. By comparing with the synchronous counterpart, we highlight the benefits of the asynchronous ABSF configuration in terms of the minimized femtocell resource expense for only a small compromise in coverage.
\end{abstract}

\section{INTRODUCTION}

With the wide utilization of smartphones and other personal mobile devices in recent years, the foremost request for cellular communications is to satisfy the unrelenting mobile broadband traffic demand. The heterogeneous network (HetNet) has been identified as a promising and cost-effective technology to meet this demand [1]. By complementing traditional macrocells with a multitude of low power small cells, such as picocell and femtocell, HetNet is able to bring the network closer to the subscribers and to most efficiently use the dimensions of space and frequency. As one of the key performance enhancement features, Third Generation Partnership Project (3GPP) has introduced the support of HetNets in LTE-Advanced (LTE-A) standardization [2].

Being an important part of LTE-A HetNets, femtocell access points, otherwise called home eNodeBs (HeNBs), are userdeployed low-power devices operating in the licensed spectrum [3], [4]. Unlike other small cells deployed by mobile operators, HeNB may selectively serve users, namely operating in closed subscriber group (CSG) mode. However, this effectively creates a coverage hole for co-channel macro user equipments (UEs) that are not CSG members of the HeNB [5]. As these victim macro UEs are not guaranteed to connect to the strongest eNodeB, they experience downlink intercell interference from nearby CSG HeNBs.

To address this challenge, time-domain enhanced intercell interference coordination (eICIC) technique was proposed [5]. In the eICIC scheme adopted in LTE release 10, the subframe-synchronized interfering eNodeB reserve resources

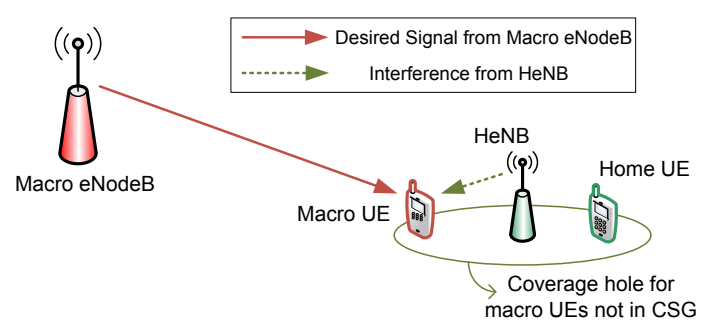

Fig. 1. The CSG HeNB scenario, where downlink strong interference and the coverage hole suffered by the macro UE not belong to nearby HeNB's CSG.

on specific subframes so that a communication link with reduced interference can be established between the serving eNodeB and the victim macro user equipment (UE). The blanked subframes are referred to as almost blank subframes (ABSFs), indicating that some basic physical channels, for example, pilot, synchronization, and broadcast channels, may still be transmitted, so as to support backward compatibility with legacy LTE release 8 UEs [6].

Although eICIC can also be applied to mitigate downlink macrocell interference for UEs in the expanded picocell regions [7], our focus in this study is limited to the abovementioned CSG HeNB scenario where macrocell UEs are experiencing downlink intercell interference from nearby CSG HeNBs..

\section{A. Asynchronous ABSF Configuration}

Depending on whether or not the common ABSF muting configuration is used among neighboring eNodeBs, the ABSF configuration can be synchronous or asynchronous [8]. In synchronous ABSF configuration, all neighboring interfering eNodeBs are coordinated to align their ABSF muting patterns. This coordinated approach greatly simplifies the optimization of eICIC into finding the optimal ABSF density, i.e., the amount of ABSFs per muting period [8]-[11].

Alternatively, each of the interfering eNodeBs in asynchronous ABSF configuration (also called dynamic blanking [12]) will independently adjust their own ABSF patterns [13], [14]. This idea was inspired by the fact that most of the interference may come from the nearest one or several 
dominant eNodeBs, while other eNodeBs contribute limited interference onto the victim UE. Though introducing a more complicated interference scenario, the asynchronous ABSF configuration provides the flexibility to accommodate different conditions among the interfering eNodeBs.

The asynchronous ABSF configuration is considered to be not appropriate for picocell range expansion scenario [8], [15]: Even when every macro eNodeB has ABSFs, the expanded picocells could still suffer from significant interference due to the uncoordinated nature among ABSF patterns of neighboring macro eNodeBs [15]. However, this conclusion can not be applied to the CSG HeNBs scenario: Firstly, the interference generated from indoor HeNBs is only influential in a limited area because of the higher pass loss exponent for indoor environments. Secondly, traffic loads and other factors could be extremely diverse across different HeNBs since the law of large numbers does not apply in range-limited femtocells [16], and thus their impacts on victim UEs can be extremely different. Furthermore, the synchronous ABSF configuration leads to the performance loss on all HeNBs, which is unreasonable for CSG HeNBs scenario, especially considering the interests of those HeNBs which are far away from any victim UEs.

\section{B. Approach and Contributions}

In this paper, we conduct analysis on the performance of asynchronous ABSF configuration implemented for the CSG HeNBs scenario, where the large-scale cellular network model is employed. In recent works, it has been shown that compared with the practical network deployment, the large-scale cellular network model with eNodeB locations drawn from a homogeneous Poisson Point Process (PPP) is as accurate as the traditional grid models [17]. More importantly, this model can provide more tractable analytical results on the coverage and throughput performance [18], [19].

The main contributions of this paper are as follows:

1) For the CSG HeNBs scenario, we provide the tractable probabilistic characterization of the coverage probability at victim macro UE, with and without the asynchronous ABSF configuration implemented in neighboring HeNBs.

2) We highlight the benefits of a strategy which only requires the highest interfering HeNB to be configured with ABSF and thus show the overall system throughput benefits from this strategy far outweigh the small increase in coverage loss on the Macro UEs.

It should be noted that similar work to evaluate the performance of large scale cellular networks with ABSF were conducted in [10], [11], [20]; however, all these existing works focused on the synchronous ABSF configuration, where all eNodeBs take the same ABSF pattern and are unable to tackle the interference environment individually and adaptively. In contrast, we draw our attention into the CSG HeNBs scenario, where asynchronous ABSF configuration is more suitable and practical.

The remainder of the paper is organised as follows. In Section II network model used in this work are introduced.
Section III provides the tractable result on the coverage performance for the two-tier femtocell networks with the asynchronous ABSF configuration implemented. Section V presents numerical results and we conclude the paper in Section VI.

\section{SySTEM MOdEL}

\section{A. Two-Tier Femtocell Large-Scale Network Model}

In our analysis, we consider a downlink LTE-A HetNet consisting of two tiers, i.e., macrocell and femtocell tiers, where spatial deployment densities, transmit powers are different across tiers. Specifically, macro and home eNodeBs are spatially distributed as independent two-dimensional (2D) PPPs $\Phi_{m}$ and $\Phi_{f}$ with the densities of $\lambda_{m}$ and $\lambda_{f}$ respectively. The transmit power of macro and home eNodeBs are respectively denoted by $P_{t x, m}$ and $P_{t x, f}$, and the same transmit power holds across each tier. HeNBs are assumed to be operated in CSG mode.

Without any loss of generality, the macro UE under analysis is assumed to be located at the origin. By assuming that this typical UE does not belong to any nearby HeNBs' subscriber groups, it is always associated to the nearest macro eNodeB, which is assume to be at $x_{0}$ and $d_{s}=\left\|x_{0}\right\|$ away from the origin.

The standard power loss propagation model is employed, in which path loss exponents are $\alpha_{m}>2$ and $\alpha_{f}>2$ for macrocell and femtocell tiers respectively, and path loss constant is $L_{0}$ at the reference distance $r_{0}=1 \mathrm{~m}$ for both tiers. For simplicity, we use $P_{m}$ and $P_{f}$ to replace the products of $L_{0}$ and the transmit powers, i.e., $P_{m}=P_{t x, m} L_{0}$ and $P_{f}=P_{t x, f} L_{0}$. We assume that the typical mobile user experiences Rayleigh fading from the serving and all the interfering eNodeBs. The fading's impact on the signal power follows the exponential distribution with the unitary mean value. The noise is assumed to be additive and constant with a variance of $\sigma^{2}$.

\section{B. Victim/Non-Victim UE}

Victim terminals are the macro UEs significantly interfered by nearby HeNBs, which are unable to establish a reliable communication link with their serving macro NodeBs. In this analysis, we assume that victim UEs are the ones which have at least one interfering HeNB with distance less than $D$ away from them, while other macro UEs are non-victim terminals.

If the typical UE is a victim interfered from nearby HeNBs, i.e., $\Phi_{f} \cap \mathcal{B}(0, D) \geqslant 1$, it will only request the nearest HeNB to apply ABSF on certain subframes to mitigate the interference ${ }^{1}$. Then, HeNB's transmit power will be attenuated by $\mu$ and become $\mu P_{f}$. Then the downlink received SINR at the typical victim UE can be expressed as

$$
\operatorname{SINR}_{v}=\frac{P_{m} h d_{s}^{-\alpha_{m}}}{I_{m}+I_{f_{r}}+I_{f_{o}}+\sigma^{2}}
$$

${ }^{1}$ We define the (closed) ball centered at $p$ and of radius $r$ as $\mathcal{B}(p, r)$, i.e., $\mathcal{B}(p, r) \triangleq\left\{m \in \mathbb{R}^{2},\|m-p\| \leqslant r\right\}$. 
in which $h$ denotes the channel fading gain from the serving macro eNodeB, $I_{m}$ is the cumulative interference from all other macro NodeBs except the one located at $x_{0}$ (the serving eNodeB for the typical UE), $I_{f_{r}}$ denotes the residual interference from the HeNB enabling ABSF, and $I_{f_{o}}$ is the sum interference power from other HeNBs. Similarly, for nonvictim typical UE, i.e., $\Phi_{f} \cap \mathcal{B}(0, D)=0$, the downlink received SINR can be expressed as

$$
\operatorname{SINR}_{n v}=\frac{P_{m} h d_{s}^{-\alpha_{m}}}{I_{m}+I_{f}+\sigma^{2}}
$$

where $I_{f}$ denotes the interference from the entire femtocell tier.

In our study, we are interested in the metric of coverage probability defined as $\mathbb{P}[\mathrm{SINR}>T]$, i.e., the probability of a target SINR $T$ (or SINR threshold) achievable at the typical UE. To derive the performance over the entire network, one can calculate the average coverage probability over the possible range of $d_{s} \in(0, \infty)$ [17]. We are also interested in determining the benefits of our approach, so we calculate the additional throughput that can be achieved by not forcing ABSFs on each HeNB but only on the major interfering HeNB. This topic will also be discussed further below.

\section{Coverage Probability}

In this section, we analyze the coverage performance over the two-tier femtocell network with asynchronous ABSF configuration implemented (only the nearest HeNB apply ABSF on certain subframes). The performance for victim and nonvictim UEs are discussed respectively as below.

\section{A. Coverage Performance for Victim UEs}

Before obtaining the coverage performance for victim UEs, firstly, let us focus on the statistic characteristics of the distance between the typical victim UE and the nearest HeNB. By defining $r_{1}$ as the distance between the typical macro UE and the nearest HeNB, the condition that the macro UE is a victim UE will be true if $r_{1} \leqslant D$, which allows us to reach the intermediate result in the following lemma.

Lemma 1: For the typical victim UE, the probability density function (PDF) of $r_{1}$ is

$$
f_{r_{1} \mid r_{1} \leqslant D}(x)=\frac{2 \pi \lambda_{f} x \exp \left(-\pi \lambda_{f} x^{2}\right)}{1-\exp \left(-\pi \lambda_{f} D^{2}\right)}, \quad \text { for } x \leqslant D .
$$

Proof: Given the condition of $r_{1} \leqslant D$, the cumulative distribution function (CDF) can be expressed as

$$
\begin{aligned}
F_{r_{1} \mid r_{1} \leqslant D}(x) & =\mathbb{P}\left[r_{1} \leqslant x \mid r_{1} \leqslant D\right] \\
& = \begin{cases}\frac{\mathbb{P}\left[r_{1} \leqslant x\right]}{\mathbb{P}\left[r_{1} \leqslant D\right]} \stackrel{(a)}{=} \frac{1-\exp \left(-\pi \lambda_{f} x^{2}\right)}{1-\exp \left(-\pi \lambda_{f} D^{2}\right)} & \text { for } x \leqslant D \\
1 & \text { for } x>D,\end{cases}
\end{aligned}
$$

in which step (a) follows the PPP's void probability, that is, the probability that the region $\mathcal{X}$ contains no point drawn from the homogeneous PPP with the density value of $\lambda$ is $\exp (-$ $\lambda A(\mathcal{X}))$, in which $A(\mathcal{X})$ is the area measure of the region $\mathcal{X}$
[21]. Hence, the PDF of $r_{1}$ can be found by differentiating the CDF, which completes the proof.

Next, we will provide one of the major results in this paper, that is, the probabilistic characterization of the downlink coverage achievable at the victim UE, with asynchronous ABSF configuration employed.

Theorem 2: Provided ABSF configuration implemented in the nearest interfering HeNB, the coverage probability for the typical victim UE with its serving eNodeB $d_{s}$ away is

$$
\begin{array}{r}
\mathbb{P}\left[\operatorname{SINR}_{v}>T\right]=\frac{\exp \left(-\pi \lambda_{m} d_{s}^{2} \rho\left(T, \alpha_{m}\right)-T d_{s}^{\alpha_{m}} \sigma^{2} / P_{m}\right)}{1-\exp \left(-\pi \lambda_{f} D^{2}\right)} \\
\cdot 2 \pi \lambda_{f} \int_{0}^{D} \exp \left(-\pi \lambda_{f} y^{2} \rho\left(\frac{T P_{f} d_{s}^{\alpha_{m}}}{P_{m} y^{\alpha_{f}}}, \alpha_{f}\right)\right) \\
\cdot \frac{y \exp \left(-\pi \lambda_{f} y^{2}\right)}{1+T d_{s}^{\alpha_{m}} \frac{\mu P_{f}}{P_{m}} y^{-\alpha_{f}}} \mathrm{~d} y
\end{array}
$$

where $\rho(T, \alpha)$ is defined as

$$
\rho(T, \alpha) \triangleq T^{2 / \alpha} \int_{T^{-2 / \alpha}}^{\infty} \frac{1}{1+u^{\alpha / 2}} \mathrm{~d} u
$$

Proof: Given the condition of $r_{1} \leqslant D$, the coverage probability can be derived as

$$
\begin{aligned}
& \mathbb{P}\left[\mathrm{SINR}_{v}>T\right] \\
& =\mathbb{P}\left[\frac{P_{m} h d_{s}^{-\alpha_{m}}}{I_{m}+I_{f_{r}}+I_{f_{o}}+\sigma^{2}}>T\right] \\
& =\mathbb{P}\left[h>T d_{s}^{\alpha_{m}} P_{m}^{-1}\left(I_{m}+I_{f_{r}}+I_{f_{o}}+\sigma^{2}\right)\right] \\
& \stackrel{(a)}{=} \exp \left(-\frac{T d_{s}^{\alpha_{m}} \sigma^{2}}{P_{m}}\right) \mathcal{L}_{I_{m}}\left(\frac{T d_{s}^{\alpha_{m}}}{P_{m}}\right) \mathcal{L}_{I_{f_{r}}+I_{f_{o}}}\left(\frac{T d_{s}^{\alpha_{m}}}{P_{m}}\right),
\end{aligned}
$$

where step $(a)$ follows the Rayleigh fading assumption, i.e., $h \sim \exp (1)$. It should be noted that $\exp \left(-T d_{s}^{\alpha_{m}} \sigma^{2} / P_{m}\right)$, $\mathcal{L}_{I_{m}}\left(T d_{s}^{\alpha_{m}} / P_{m}\right)$, and $\mathcal{L}_{I_{f_{r}}+I_{f_{o}}}\left(T d_{s}^{\alpha_{m}} / P_{m}\right)$ respectively represent the impact of the noise, the macrocell interference, and the femtocell interference. $\mathcal{L}_{I_{m}}(\cdot)$ and $\mathcal{L}_{I_{f_{r}}+I_{f_{o}}}(\cdot)$ are the Laplace transforms of the interference values $I_{m}$ and $I_{f_{r}}+I_{f_{o}}$ respectively.

The Laplace transform $\mathcal{L}_{I_{m}}\left(T d_{s}^{\alpha_{m}} / P_{m}\right)$ has been derived in [17], that is,

$$
\mathcal{L}_{I_{m}}\left(\frac{T d_{s}^{\alpha_{m}}}{P_{m}}\right)=\exp \left(-\pi \lambda_{m} d_{s}^{2} \rho\left(T, \alpha_{m}\right)\right) .
$$

On the other hand, $\mathcal{L}_{I_{f_{r}}+I_{f_{o}}}\left(T d_{s}^{\alpha_{m}} / P_{m}\right)$ can be derived as,

$$
\begin{aligned}
& \mathcal{L}_{I_{f_{r}}+I_{f_{o}}}\left(\frac{T d_{s}^{\alpha_{m}}}{P_{m}}\right) \\
& =\mathbb{E}_{I_{f_{r}}+I_{f_{o}}}\left[\exp \left(-\frac{T d_{s}^{\alpha_{m}}\left(I_{f_{r}}+I_{f_{o}}\right)}{P_{m}}\right)\right] \\
& \stackrel{(b)}{=} \int_{0}^{D} \mathcal{L}_{I_{f_{r}} \mid r_{1}=y}\left(\frac{T d_{s}^{\alpha_{m}}}{P_{m}}\right) \mathcal{L}_{I_{f_{o}} \mid r_{1}=y}\left(\frac{T d_{s}^{\alpha_{m}}}{P_{m}}\right) f_{r_{1} \mid r_{1} \leqslant D}(y) \mathrm{d} y,
\end{aligned}
$$

where step $(b)$ is calculated by averaging over the whole range of possible $r_{1}$, i.e., $r_{1} \in(0, D]$. As described in Section II-B, 
only the nearest HeNB will apply ABSF configuration, therefore the impact from its residual interference can be derived as

$$
\begin{aligned}
\mathcal{L}_{I_{f_{r}} \mid r_{1}=y}\left(\frac{T d_{s}^{\alpha_{m}}}{P_{m}}\right) & =\mathbb{E}_{I_{f_{r}} \mid r_{1}=y}\left[\exp \left(-\frac{T d_{s}^{\alpha_{m}} I_{f_{r}}}{P_{m}}\right)\right] \\
& \stackrel{(c)}{=} \mathbb{E}_{h_{s}}\left[\exp \left(-\frac{T d_{s}^{\alpha_{m}} h_{s} \mu P_{f} y^{-\alpha_{f}}}{P_{m}}\right)\right] \\
& \stackrel{(d)}{=} \frac{1}{1+T d_{s}^{\alpha_{m}} h_{s} y^{-\alpha_{f}} \frac{\mu P_{f}}{P_{m}}}
\end{aligned}
$$

where $h_{s}$ in step $(c)$ is the channel fading gain from the nearest $\mathrm{HeNB}$, and the expectation is conducted over $h_{s}$. Following the Rayleigh fading assumption for $h_{s}$, step $(d)$ can be obtained.

For the sum interference power from other HeNBs, we can have

$$
\begin{aligned}
& \mathcal{L}_{I_{f_{o}} \mid r_{1}=y}\left(\frac{T d_{s}^{\alpha_{m}}}{P_{m}}\right)=\mathbb{E}_{I_{f_{o}} \mid r_{1}=y}\left[\exp \left(-\frac{T d_{s}^{\alpha_{m}} I_{f_{o}}}{P_{m}}\right)\right] \\
& \stackrel{(e)}{=} \mathbb{E}_{\Phi_{f} \mid r_{1}=y}\left[\prod_{x \in \Phi_{f} \backslash\left\{x_{0}\right\}} \mathbb{E}_{h_{x}}\left[\exp \left(-T d_{s}^{\alpha_{m}} \frac{P_{f}}{P_{m}} h_{x}\|x\|^{-\alpha_{f}}\right)\right]\right] \\
& \stackrel{(f)}{=} \exp \left(-\lambda_{f} \int_{\mathbb{R}^{2} \backslash \mathcal{B}(0, y)}\left(1-\mathbb{E}_{h_{x}}\left[\exp \left(-T d_{s}^{\alpha_{m}} \frac{P_{f}}{P_{m}} h_{x}\|x\|^{-\alpha_{f}}\right)\right]\right) \mathrm{d} x\right) \\
& \stackrel{(g)}{=} \exp \left(-2 \pi \lambda_{f} \int_{y}^{\infty}\left(1-\frac{1}{1+T d_{s}^{\alpha_{m}} \frac{P_{f}}{P_{m}} t^{-\alpha_{f}}}\right) t \mathrm{~d} t\right) \\
& =\exp \left(-\pi \lambda_{f} y^{2} \rho\left(\frac{T P_{f} d_{s}^{\alpha_{m}}}{P_{m} y^{\alpha_{f}}}, \alpha_{f}\right)\right),
\end{aligned}
$$

in which $h_{x}$ in step $(e)$ is the channel fading gain from the HeNB at the location $x$, and step $(e)$ is based upon the independence among different elements of $\left\{h_{x}: x \in\right.$ $\left.\Phi_{f} \backslash\left\{x_{0}\right\}\right\}$ and their further independence from the PPP $\Phi_{f}$. Step $(f)$ is derived because of the result for PPP's probability generating functional (PGFL) [21], and step $(g)$ follows the Rayleigh fading assumption for $h_{x}$. Through mathematical manipulation, the integral can be expressed in the form of function $\rho(\cdot)$, which has been widely used for similar problems [22] [17].

By substituting (10) and (11) into (9), we can obtain the $\mathcal{L}_{I_{f_{r}}+I_{f_{o}}}\left(T d_{s}^{\alpha_{m}} / P_{m}\right)$, which can be combined with (7), (8), and the PDF of $r_{1}$ provided in Lemma 1, to reach the coverage probability result in (5). Till here, the proof has been completed.

\section{B. Coverage Performance for Non-Victim UEs}

Given the condition that the UE is a non-victim terminal, we can determine that the distance between the typical UE and the nearest HeNB should be larger than $D$, i.e., $r_{1}>D$. Then, based on that, the non-victim UE's downlink coverage performance is possible to calculate. It should be noted that ABSF configuration will not be initialized in this case.

Theorem 3: The coverage probability achievable at the typical non-victim UE with its serving eNodeB $d_{s}$ away can be expressed as

$$
\begin{gathered}
\mathbb{P}\left[\operatorname{SINR}_{n v}>T\right]=\exp \left(-\pi \lambda_{m} d_{s}^{2} \rho\left(T, \alpha_{m}\right)-T d_{s}^{\alpha_{m}} \sigma^{2} / P_{m}\right) \\
\cdot \exp \left(\pi \lambda _ { f } \left(\frac{\omega D^{2}}{D^{\alpha_{f}}+\omega}-\omega^{\frac{2}{\alpha_{f}}} \Gamma\left(1+\frac{2}{\alpha_{f}}\right) \Gamma\left(1-\frac{2}{\alpha_{f}}\right)\right.\right. \\
\left.\left.+\frac{\omega D^{2-\alpha_{f}}}{\left(1-\frac{2}{\alpha_{f}}\right)\left(1+\frac{\omega}{D^{\alpha_{f}}}\right)^{2}} \cdot{ }_{2} \mathrm{~F}_{1}\left(1,2 ; 2+\frac{2}{\alpha_{f}} ; \frac{1}{1+\frac{\omega}{D^{\alpha_{f}}}}\right)\right)\right)
\end{gathered}
$$

where the constant $\omega$ is defined as

$$
\omega=T d_{s}^{\alpha_{m}} \frac{P_{f}}{P_{m}},
$$

$\Gamma(x)$ is the standard gamma function, and ${ }_{2} \mathrm{~F}_{1}(\cdot)$ is the Gauss hypergeometric function.

Proof: Given the conditoin of $r_{1}>D$, the coverage probability can be derived as

$$
\begin{aligned}
& \mathbb{P}\left[\operatorname{SINR}_{n v}>T\right] \\
& =\mathbb{P}\left[\frac{P_{m} h d_{s}^{-\alpha_{m}}}{I_{m}+I_{f}+\sigma^{2}}>T\right] \\
& =\mathbb{P}\left[h>T d_{s}^{\alpha_{m}} P_{m}^{-1}\left(I_{m}+I_{f}+\sigma^{2}\right)\right] \\
& \stackrel{(a)}{=} \exp \left(-\frac{T d_{s}^{\alpha_{m}} \sigma^{2}}{P_{m}}\right) \mathcal{L}_{I_{m}}\left(\frac{T d_{s}^{\alpha_{m}}}{P_{m}}\right) \mathcal{L}_{I_{f}}\left(\frac{T d_{s}^{\alpha_{m}}}{P_{m}}\right),
\end{aligned}
$$

where step $(a)$ also follows the Rayleigh fading assumption, and $\mathcal{L}_{I_{m}}\left(T d_{s}^{\alpha_{m}} / P_{m}\right)$ is provided in (8) already. For the sum interference power from femtocell tier, we can have

$$
\begin{aligned}
& \mathcal{L}_{I_{f}}\left(\frac{T d_{s}^{\alpha_{m}}}{P_{m}}\right)=\mathbb{E}_{I_{f}}\left[\exp \left(-\frac{T d_{s}^{\alpha_{m}} I_{f}}{P_{m}}\right)\right] \\
& \stackrel{(b)}{=} \mathbb{E}_{\Phi_{f}}\left[\prod_{x \in \Phi_{f} \backslash \mathcal{B}(0, D)} \mathbb{E}_{h_{x}}\left[\exp \left(-T d_{s}^{\alpha_{m}} \frac{P_{f}}{P_{m}} h_{x}\|x\|^{-\alpha_{f}}\right)\right]\right] \\
& \stackrel{(c)}{=} \exp \left(-\lambda_{f} \int_{\mathbb{R}^{2} \backslash \mathcal{B}(0, D)} \quad\left(1-\mathbb{E}_{h_{x}}\left[\exp \left(-T d_{s}^{\alpha_{m}} \frac{P_{f}}{P_{m}} h_{x}\|x\|^{-\alpha_{f}}\right)\right]\right) \mathrm{d} x\right) \\
& \stackrel{(d)}{=} \exp \left(\pi \lambda _ { f } \left[\frac{\omega D^{2}}{D^{\alpha_{f}}+\omega}-\omega^{\frac{2}{\alpha_{f}}} \Gamma\left(1+\frac{2}{\alpha_{f}}\right) \Gamma\left(1-\frac{2}{\alpha_{f}}\right)\right.\right. \\
& +\frac{\omega D^{2-\alpha_{f}}}{\left(1-\frac{2}{\alpha_{f}}\right)\left(1+\frac{\omega}{D^{\alpha_{f}}}\right)^{2}} \cdot{ }_{2} \mathrm{~F}_{1}\left(1,2 ; 2+\frac{2}{\alpha_{f}} ; \frac{1}{\left.\left.\left.1+\frac{\omega}{D^{\alpha_{f}}}\right)\right]\right)}\right.
\end{aligned}
$$

in which step $(b)$ is based upon the independence among different elements of $\left\{h_{x}: x \in \Phi_{f} \backslash\left\{x_{0}\right\}\right\}$ and their further independence from the PPP $\Phi_{f}$, and Step $(c)$ is also based on PPP's PGFL. Based upon the result in [23], step $(d)$ can be obtained.

By substituting (8) and (15) into (14), the coverage probability in (12) can be reached, which completes the proof.

\section{Coverage Performance for a Randomly Chosen UEs}

For a randomly chosen UE with the distance $d_{s}$ from the serving macro eNodeB, the coverage performance can be 
obtained by combining the results for victim and non-victim UEs.

Corollary 4: The coverage probability for the randomly chosen typical macro UE with its serving eNodeB $d_{s}$ away is

$$
\begin{aligned}
\mathbb{P}[\operatorname{SINR}>T]= & \mathbb{P}\left[\operatorname{SINR}_{v}>T\right] \cdot\left[1-\exp \left(-\pi \lambda_{f} D^{2}\right)\right] \\
& +\mathbb{P}\left[\operatorname{SINR}_{n v}>T\right] \cdot \exp \left(-\pi \lambda_{f} D^{2}\right),
\end{aligned}
$$

where $\mathbb{P}\left[\operatorname{SINR}_{v}>T\right]$ and $\mathbb{P}\left[\operatorname{SINR}_{n v}>T\right]$ are the coverage probabilities achievable at the typical victim UE and nonvictim UE provided by Theorem 2 and Theorem 3 .

Proof: The coverage probability can be expressed as

$$
\begin{aligned}
\mathbb{P}[\operatorname{SINR}>T]=\mathbb{P}\left[\operatorname{SINR}_{v}>T\right] \cdot \mathbb{P}\left[\Phi_{f} \cap \mathcal{B}(0, D) \geqslant 1\right] \\
+\mathbb{P}\left[\operatorname{SINR}_{n v}>T\right] \cdot \mathbb{P}\left[\Phi_{f} \cap \mathcal{B}(0, D)=0\right] .
\end{aligned}
$$

Based on the fact that $\Phi_{f}$ is PPP distributed, we can follow the PPP's void probability to obtain $\mathbb{P}\left[\Phi_{f} \cap \mathcal{B}(0, D) \geqslant 1\right]$ and $\mathbb{P}\left[\Phi_{f} \cap \mathcal{B}(0, D)=0\right]$, i.e.,

$$
\mathbb{P}\left[\Phi_{f} \cap \mathcal{B}(0, D) \geqslant 1\right]=1-\exp \left(-\pi \lambda_{f} D^{2}\right),
$$

and

$$
\mathbb{P}\left[\Phi_{f} \cap \mathcal{B}(0, D)=0\right]=\exp \left(-\pi \lambda_{f} D^{2}\right),
$$

which helps us to complete the proof.

\section{RESOURCE EXPENSE OF ASYNCHRONOUS ABSF CONFIGURATION}

Here, we use the average number of HeNBs expected to participate asynchronous $\mathrm{ABSF}$ configuration, i.e., $N$, to measure the resource expense. Obviously, $N$ is the function of $D$, which obeys the following relationship.

Proposition 5: The average number of HeNBs participating ABSF configuration (muting HeNBs)

$$
N(D)=1-\exp \left(-\pi \lambda_{f} D^{2}\right)
$$

Proof: Based on the fact that only the nearest HeNB participates in the ABSF configuration, we can have

$$
\begin{aligned}
N(D) & =\mathbf{1}\left[\Phi_{f} \cap \mathcal{B}(0, D)\right] \\
& =\mathbb{P}\left[\Phi_{f} \cap \mathcal{B}(0, D) \geqslant 1\right],
\end{aligned}
$$

where $\mathbf{1}[\varphi]$ is the indicator function, having the value of 1 if the event $\varphi$ is not void and the value of 0 otherwise. Then, (20) can be obtained easily.

\section{NumERICAL RESULTS}

In this section, we present the numerical results for the coverage performance for a two-tier LTE-A femtocell network with asynchronous ABSF configuration implemented. Specifically, the macro eNodeBs at high power level, i.e., $P_{t x, 1}=46 \mathrm{dBm}$, are co-deployed with lower power level HeNBs with $P_{t x, 2}=20 \mathrm{dBm}$. The densities of macro and home eNodeBs are respectively $\lambda_{m}=1$ and $\lambda_{f}=40$ per square $\mathrm{km}$ in all numerical results. The path loss model utilized here has the parameters of $L_{0}=-34 \mathrm{~dB}, \alpha_{m}=3.5$ and

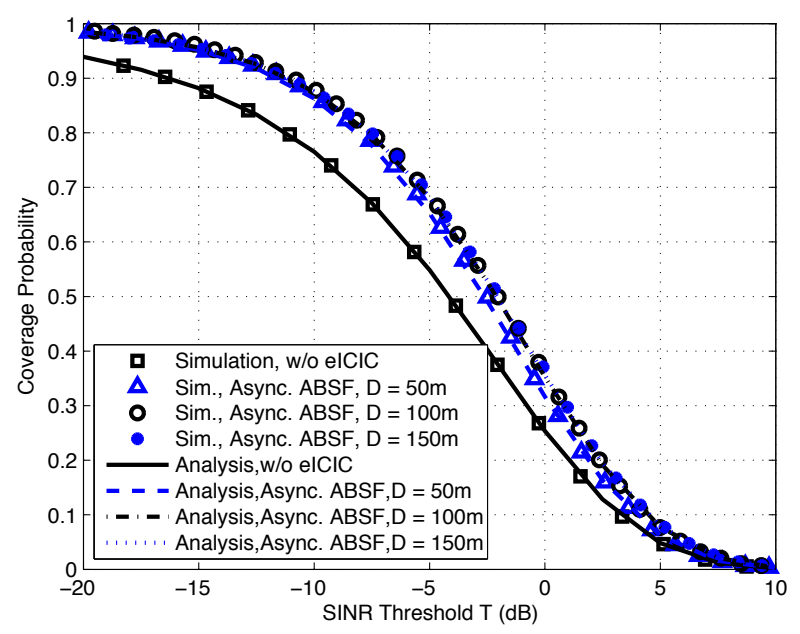

Fig. 2. Coverage probability for a typical macro UE with $d_{s}=500 \mathrm{~m}$.

$\alpha_{f}=4$. The thermal noise power is $\sigma^{2}=-104 \mathrm{dBm}$. It is assumed that no residual interference is left after ABSF, i.e., $\mu=0$. Monte Carlo simulations are also conducted to compare with our analysis for the purpose of model validation.

Fig. 2 show the coverage probability over different SINR thresholds. It can be concluded that the analysis well matches the simulation results and demonstrated that asynchronous ABSF configuration plays a significant role in enhancing the coverage probability at the typical user. By increasing the distance $D$, the typical UE has more chance to be claimed as a victim terminal, however continuing the increase of $D$ (e.g. $D=100,150 \mathrm{~m}$ ) only pays back a minor improvement in coverage probability.

As discussed previously, it is possible for more HeNBs to apply ABSF configuration, which would reduce interference and thus improve coverage performance. The extreme case is synchronous ABSF configuration in which all nearby HeNBs in $\mathcal{B}(0, D)$ are coordinated to be ABSF synchronous. However, reserving resources (with the use of the ABSF) for the victim UEs means a network throughput (resource) loss on the donor HeNBs.

Fig. 3 and 4 show the trade-off between femtocell resource utilization (related to system throughput) and coverage probability for asynchronous ABSF configuration with different $D$. The performance of different synchronous ABSF schemes are also demonstrated. It can be seen that asynchronous ABSF configuration minimizes the resource expense for interference avoidance and thus increases the system throughput performance. The figures highlight that increasing the radius above $50 \mathrm{~m}$ brings little in terms of coverage probability while reducing the available femtocell resources. This therefor highlights that a radius of $50 \mathrm{~m}$ brings the most benefits in terms of coverage while minimizing the network throughput reduction.

\section{CONCLUSION}

In this paper we studied the performance of asynchronous ABSF configuration in a two-tier femtocell HetNet. With 


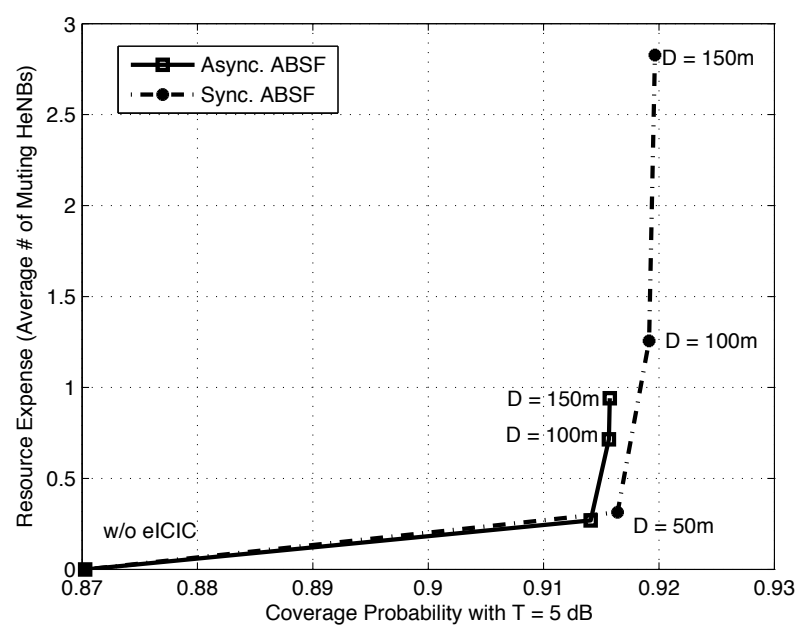

Fig. 3. The tradeoff between coverage probability and resource expense, for a typical macro UE with $d_{s}=100 \mathrm{~m}, T=5 \mathrm{~dB}$.

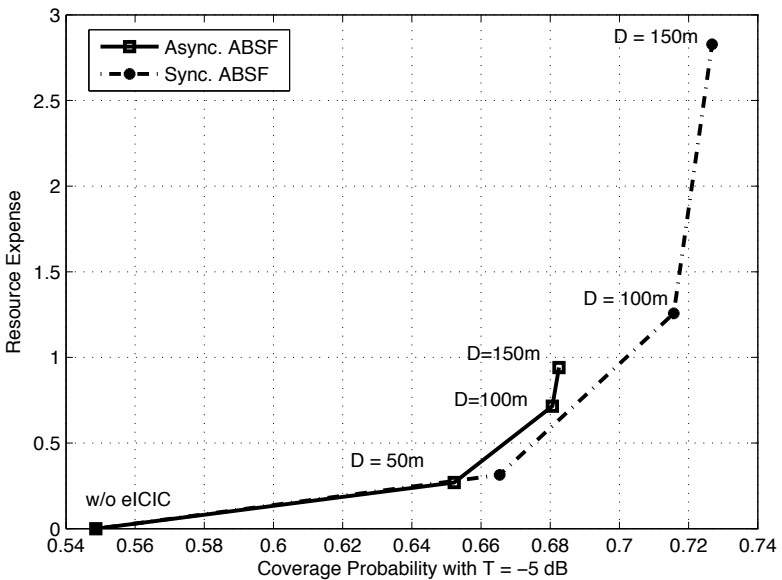

Fig. 4. The tradeoff between coverage probability and resource expense, for a typical macro UE with $d_{s}=500 \mathrm{~m}, T=-5 \mathrm{~dB}$.

a stochastic geometric framework we showed the coverage probability via analysis and verified with simulation. We highlighted the trade-off between system throughput and coverage probability, and demonstrated that by removing the main interfering femtocell with an ABSF, minimal system resource is lost while coverage probability can still be well maintained. This trade-off needs to be taken into account when considering interference avoidance strategies. Analyzing this trade-off in terms of the overall network throughput, and broadening the asynchronous ABSF configuration with multiple major interferers are left for future work.

\section{REFERENCES}

[1] A. Ghosh, N. Mangalvedhe, R. Ratasuk, B. Mondal, M. Cudak, E. Visotsky, T. A. Thomas, J. G. Andrews, P. Xia, H.-S. Jo, H. S. Dhillon, and T. D. Novlan, "Heterogeneous cellular networks: From theory to practice,” IEEE Commun. Mag., vol. 50, no. 6, pp. 54-64, 2012.
[2] A. Damnjanovic, J. Montojo, Y. Wei, T. Ji, T. Luo, M. Vajapeyam, T. Yoo, O. Song, and D. Malladi, "A survey on 3GPP heterogeneous networks," IEEE Wireless Commun., vol. 18, no. 3, pp. 10-21, 2011.

[3] J. G. Andrews, H. Claussen, M. Dohler, S. Rangan, and M. C. Reed, "Femtocells: Past, present, and future," IEEE J. Select. Areas Commun., vol. 30, no. 3, pp. 497-508, Apr. 2012.

[4] Z. Shi, H. Wang, M. Zhao, and M. C. Reed, "An uplink analytical model for two-tiered 3G femtocell networks," in Proc. 8th Int'l Symp. on Modeling and Optimization in Mobile, Ad Hoc, and Wireless Networks (WiOpt'10), Avignon, France, May 2010, pp. 367-372.

[5] D. Lopez-Perez, I. Guvenc, G. d. 1. Roche, M. Kountouris, T. Q. S. Quek, and J. Zhang, "Enhanced intercell interference coordination challenges in heterogeneous networks," IEEE Wireless Commun., vol. 18, no. 3, pp. 22-30, 2011.

[6] K. Okino, T. Nakayama, C. Yamazaki, H. Sato, and Y. Kusano, "Pico cell range expansion with interference mitigation toward LTE-Advanced heterogeneous networks," in Proc. IEEE Int'l Conf. on Commun. (ICC'11), Kyoto, Japan, June 2011, pp. 1-5.

[7] H. Holma and A. Toskala, LTE-Advanced: 3GPP Solution for IMTAdvanced. New York, NY: John Wiley \& Sons Ltd., 2012.

[8] J. Pang, J. Wang, D. Wang, G. Shen, Q. Jiang, and J. Liu, "Optimized time-domain resource partitioning for enhanced inter-cell interference coordination in heterogeneous networks," in Proc. 2012 IEEE Wireless Commun. and Networking Conference Workshops (WCNCW'12), Paris, France, Apr. 2012, pp. 1613-1617.

[9] S. Vasudevan, R. Pupala, and K. Sivanesan, "Dynamic eICIC - A proactive strategy for improving spectral efficiencies of heterogeneous LTE cellular networks by leveraging user mobility and traffic dynamics," IEEE Trans. Wireless Commun., 2013, to be published.

[10] M. Čierny, H. Wang, R. Wichman, Z. Ding, and C. Wijting. On number of almost blank subframes in heterogeneous cellular networks. [Online]. Available: http://arxiv.org/abs/1304.2269

[11] S. Singh and J. G. Andrews. Joint resource partitioning and offloading in heterogeneous cellular networks. [Online]. Available: http://arxiv.org/abs/1304.2269

[12] 3GPP R1-112510, "Discussion on the performance aspects of CoMP and eICIC in HetNet," Aug. 2011.

[13] 3GPP R1-112441, "Coordinated scheduling (CS) schemes with low power RRH: Details and Phase-2 evaluations results," Aug. 2011.

[14] M. I. Kamel and K. M. F. Elsayed. ABSF offsetting and optimal resource partitioning for eICIC in LTE-Advanced: Proposal and analysis using a Nash bargaining approach. [Online]. Available: http://4gpp-project.net/

[15] 3GPP R1-100142, "System performance of heterogeneous networks with range expansion," Jan. 2010.

[16] J. G. Andrews, "Seven ways that HetNets are a cellular paradigm shift," IEEE Commun. Mag., vol. 51, no. 3, pp. 136-144, 2013.

[17] J. G. Andrews, F. Baccelli, and R. K. Ganti, "A tractable approach to coverage and rate in cellular networks," IEEE Trans. Commun., vol. 59, no. 11 , pp. 3122-3134, Nov. 2011.

[18] M. Haenggi, J. G. Andrews, F. Baccelli, O. Dousse, and M. Franceschetti, "Stochastic geometry and random graphs for the analysis and design of wireless networks," IEEE J. Select. Areas Commun., vol. 27, no. 7, pp. 1029-1046, Sept. 2009.

[19] H. Wang, X. Zhou, and M. C. Reed, "Coverage and throughput analysis with a non-uniform small cell deployment," IEEE Trans. Wireless Commun., 2014, to be published.

[20] C. H. M. de Lima, M. Bennis, and M. Latva-aho. Statistical analysis of self-organizing networks with biased cell association and interference avoidance. [Online]. Available: http://arxiv.org/abs/1301.3601

[21] D. Stoyan, W. S. Kendall, and J. Mecke, Stochastic Geometry and its Applications, 2nd ed. New York, NY: John Wiley \& Sons Ltd., 1995.

[22] S. Singh, H. S. Dhillon, and J. G. Andrews. Offloading in heterogeneous networks: Modeling, analysis and design insights. [Online]. Available: http://arxiv.org/abs/1208.1977

[23] X. Zhou, R. K. Ganti, J. G. Andrews, and A. Hjørungnes, "On the throughput cost of physical layer security in decentralized wireless networks," IEEE Trans. Wireless Commun., vol. 10, no. 8, pp. 27642775, Aug. 2011. 